\title{
NONREALIZABILITY OF SUBALGEBRAS OF $\mathfrak{A}^{*}$
}

\author{
STANLEY O. KOCHMAN
}

(Communicated by Frederick R. Cohen)

\begin{abstract}
At the prime two, the dual of the Steenrod algebra is a polynomial algebra in generators $\xi_{n}, n \geq 1$. The Eilenberg-Mac Lane spectrum $K\left(Z_{2}\right)$ has homology $Z_{2}\left[\xi_{n} \mid n \geq 1\right]$, the Brown-Peterson spectrum BP has homology $Z_{2}\left[\xi_{n}^{2} \mid n \geq 1\right]$, and the symplectic Thom spectrum MSp has homology $Z_{2}\left[\xi_{n}^{4} \mid n \geq 1\right] \otimes \mathfrak{S}$. In this paper, we show that there is no spectrum $B_{k}$ with $H_{*} B_{k}=Z_{2}\left[\xi_{n}^{2^{k}} \mid n \geq 1\right]$ for $k \geq 2$.
\end{abstract}

In this paper, all spectra are localized at the prime two, and all coefficients are $Z_{2}$. All our spectra $E$ have units $\mu: S \rightarrow E$, and all our ring spectra have a homotopy unit, are homotopy associative, and are homotopy commutative. Let $\mathfrak{A}^{*}=Z_{2}\left[\xi_{n} \mid n \geq 1\right]$ denote the dual of the Steenrod algebra. Recall $[2,7]$ that as algebras and $\mathfrak{A}^{*}$-comodules, $H_{*} K Z_{2}=Z_{2}\left[\xi_{n} \mid n \geq 1\right], H_{*} \mathrm{BP}=Z_{2}\left[\xi_{n}^{2} \mid n \geq 1\right]$, and $H_{*} \mathrm{MSp}=Z_{2}\left[\xi_{n}^{4} \mid n \geq 1\right] \otimes \mathfrak{S}$, where $\mathfrak{S}=Z_{2}\left[V_{m} \mid m \neq 2^{q}-1\right]$. The $V_{m}$ are $\mathfrak{A}^{*}$-primitive elements of degree $4 m$. In this paper we show that for $k \geq 2$ there is no ring spectrum $B_{k}$ such that $H_{*} B_{k}=Z_{2}\left[\xi_{n}^{2^{k}} \mid n \geq 1\right]$ as algebras and $\mathfrak{A}^{*}$-comodules. For $k \geq 4$, we prove the stronger result that there is no spectrum $\mathfrak{B}_{k}$ such that $H_{*} \mathfrak{B}_{k}=Z_{2}\left[\xi_{n}^{2^{k}} \mid n \geq 1\right] \otimes \mathfrak{S}_{k}$ as $\mathfrak{A}^{*}$-comodules, where $\mathfrak{S}_{k}$ is a set of $\mathfrak{A}^{*}$-primitive elements. Of course, MSp is an example of a $\mathfrak{B}_{2}$. We cannot determine whether any $\mathfrak{B}_{3}$ exist. If spectra of the type $\mathfrak{B}_{k}, k \geq 3$, had existed, they would have defined generalized Adams spectral sequences which would have been efficient methods for computing $\pi_{*}^{S}$. (For example, see [6] for a description of the MSp-Adams-Novikov spectral sequence for $\pi_{*}^{S}$.)

Assume that $B_{k}$ exists with $k \geq 2$. Consider the Adams spectral sequence:

$$
E_{2}^{n, t}=\operatorname{Ext}_{\mathfrak{A}}^{t}\left(H^{*} B_{k}, Z_{2}\right)_{n} \Rightarrow \pi_{*} B_{k} .
$$

Note that since $B_{k}$ may not be a ring spectrum, the Adams spectral sequence (A) may not have a multiplicative structure. However, $H_{*} B_{k}=Z_{2}\left[\xi_{n}^{2^{k}} \mid n \geq 1\right]$ is

Received by the editors March 9, 1990.

1980 Mathematics Subject Classification (1985 Revision). Primary 55P42; Secondary 55N22, $55 \mathrm{~S} 10,55 \mathrm{~T} 15$.

This research was partially supported by a grant from the Natural Sciences and Engineering Research Council of Canada. 
a sub-Hopf algebra of $\mathfrak{A}^{*}$. Let $T(k)$ denote the truncated polynomial algebra $Z_{2}\left[\xi_{n} \mid n \geq 1\right] /\left(\xi_{n}^{2^{k}} \mid n \geq 1\right)$. By the change of rings theorem of A. Liulevicius [7]

$$
\begin{aligned}
\operatorname{Ext}_{\mathfrak{A}}\left(H^{*} B_{k}, Z_{2}\right) & \cong \operatorname{Cotor}_{\mathfrak{A}^{*}}\left(H_{*} B_{k}, Z_{2}\right) \\
& =\operatorname{Cotor}_{\mathfrak{A}}\left(Z_{2}\left[\xi_{n}^{2^{k}} \mid n \geq 1\right], Z_{2}\right) \cong \operatorname{Cotor}_{T(k)}\left(Z_{2}, Z_{2}\right) .
\end{aligned}
$$

We use the May spectral sequence [8] to study $\operatorname{Cotor}_{T(k)}\left(Z_{2}, Z_{2}\right)$ :

$$
{ }_{M} E_{2}^{n, t}=\operatorname{Cotor}_{E^{0} T(k)}^{t}\left(Z_{2}, Z_{2}\right)_{n} \Rightarrow \operatorname{Cotor}_{T(k)}^{t}\left(Z_{2}, Z_{2}\right)_{n} .
$$

$E^{0} T(k)$ is the associated graded algebra of $T(k)$ induced by the coproduct filtration. Let $\mathfrak{C}^{*} T(k)$ denote the cobar construction of $T(k)$. The following lemma describes a DGA algebra ${ }_{M} E_{1}$ whose homology is ${ }_{M} E_{2}$.

Lemma 1. Let $h_{n j}=\left[\xi_{n}^{2^{j}}\right] \in \mathfrak{C}^{2^{n+j}-2^{j}-1,1} T(k)$. Then

$$
{ }_{M} E_{1}=Z_{2}\left[h_{n j} \mid n \geq 1 \text { and } k>j \geq 0\right] \text {. }
$$

Moreover, $d_{1}\left(h_{n j}\right)=\sum_{t=1}^{k-j-1} h_{n-t, j+t} h_{t j}$ for $0 \leq j \leq k-2$, and $d_{1}\left(h_{n, k-1}\right)=0$.

Proof. Let $E(S), P(S), \Gamma(S)$ denote the exterior algebra, polynomial algebra, and divided polynomial algebra, respectively, on the set $S$, and let $V(\mathfrak{L})$ denote the universal enveloping algebra of the restricted Lie algebra $\mathfrak{L}$ [10]. Let $\xi_{n j}=$ $\left\{\xi_{n}^{2^{j}}\right\} \in E^{0} T(k)$. Then $E^{0} T(k)=E\left(\xi_{n j} \mid n \geq 1, k>j \geq 0\right)$ with $\widetilde{\Delta}\left(\xi_{n j}\right)=$ $\sum_{t=1}^{k-j-1} \xi_{n-t, t+j} \otimes \xi_{t j}$. Thus, $\left[E^{0} T(k)\right]^{*}=V(\mathfrak{L})$, where $\mathfrak{L}$ is the restricted Lie algebra with $Z_{2}$-basis $\left\{\xi_{n j}^{*} \mid n \geq 1, k>j \geq 0\right\}$, zero restriction, and Lie bracket $\left[\xi_{m i}^{*}, \xi_{n j}^{*}\right]$ equal to $\xi_{m+n, i}^{*}, \xi_{m+n, j}^{*}, 0$ if $m+i=j, n+j=i, m+i \neq j$ and $n+j \neq i$, respectively. By [9, Remark 10], there is a differential on the $Z_{2}$-coalgebra $X=\Gamma(s \mathfrak{L}) \otimes V(\mathfrak{L})$ making $X$ a free $\left[E^{0} T(k)\right]^{*}$-resolution of $Z_{2}$. Thus,

$$
\begin{aligned}
{ }_{M} E_{2} & =H_{*}\left[\operatorname{Hom}_{V(\mathfrak{L})}\left(X, Z_{2}\right)\right] \\
& \cong H_{*}\left[\operatorname{Hom}_{Z_{2}}\left(\Gamma(s \mathfrak{L}), Z_{2}\right)\right] \cong H_{*} P\left[(s \mathfrak{L})^{*}\right] \\
& \cong H_{*}\left(Z_{2}\left[h_{n j} \mid n \geq 1, k>j \geq 0\right]\right),
\end{aligned}
$$

where $h_{n j}=\left(s \xi_{n j}^{*}\right)^{*}$ is represented by $\left[\xi_{n}^{2^{j}}\right]$ in the cobar construction. Clearly $d_{1}$, being induced by $\widetilde{\Delta}\left(\xi_{n j}\right)$, is given by $d_{1}\left(h_{n j}\right)=\sum_{t=1}^{k-j-1} h_{n-t, j+t} h_{t j}$ for $0 \leq j \leq k-1$. The situation is analogous to that of [8, Chapter $2, \S 3]$ and [5, $\S 1]$.

Lemma 2. For $k \geq 4$, there is a nonzero element $h_{0} h_{k-1}^{2} \in E_{2}^{2^{k}-2,3}$.

Proof. Clearly $h_{10} h_{1, k-1}^{2}$ is a nonzero infinite cycle in ${ }_{M} E_{1}^{2^{k}-2,3}$. So ${ }_{M} E_{1}^{2^{k}-1,2}$ $=Z_{2}\left\{h_{20} h_{k-1,1}, h_{11} h_{k 0}\right\}$, and $d_{1}\left(h_{20} h_{k-1,1}\right), d_{1}\left(h_{11} h_{k 0}\right)$ contains $h_{20} h_{21} h_{k-3,3}$, $h_{11} h_{30} h_{k-3,3}$, respectively, as a nonzero summand because $k \geq 4$. Thus, 
${ }_{M} E_{2}^{2^{k}-1,2}=0, h_{10} h_{1, k-1}^{2}$ is nonzero in ${ }_{M} E_{\infty}^{2^{k}-2,3}$ and defines a nonzero element $h_{0} h_{k-1}^{2}$ of $E_{2}^{2^{k}-2,3}$.

Theorem 3. Spectra $\mathfrak{B}_{k}$ and $B_{k}$ do not exist for $k \geq 4$.

Proof. Consider the unit map $\mu: S \rightarrow B_{k}$. Let the $h_{k}$ be represented by the $h_{1, k}$ in the May spectral sequences. By Adams [1], $d_{2}\left(h_{k}\right)=h_{0} h_{k-1}^{2}$ for $k \geq 4$ in the Adams spectral sequence for $\pi_{*} S$ :

$$
E_{2}^{n, t}=\operatorname{Ext}_{\mathfrak{A}}^{t}\left(Z_{2}, Z_{2}\right)_{n} \Rightarrow \pi_{*} S .
$$

$\mu$ induces a map of spectral sequences $\left\{\mu_{r}\right\}$ between the Adams spectral sequences (C) and (A). Observe that $\mu_{2}$ is induced by the map of algebras $\mu_{*}: Z_{2} \rightarrow H_{*} B_{k}=Z_{2}\left[\xi_{m}^{2^{k}} \mid m \geq 1\right]$, and thus $\mu_{2}$ is an algebra homomorphism. Clearly $\mu_{2}\left(h_{i}\right)=h_{i}$ for $0 \leq i \leq k-1$, and $\mu_{2}\left(h_{k}\right)=0$. Thus, $h_{0} h_{k-1}^{2}$ must be zero in $E_{2}$ of the Adams spectral sequence (A), contradicting Lemma 2. Therefore, $B_{k}$ cannot exist for $k \geq 4$. The $E_{2}$-term of the Adams spectral sequence for $\pi_{*} \mathfrak{B}_{k}$ equals $\operatorname{Cotor}_{T(k)}\left(Z_{2}, Z_{2}\right) \otimes \mathfrak{S}_{k}$. Thus, the above argument, with the Adams spectral sequence for $\pi_{*} \mathfrak{B}_{k}$ replacing the Adams spectral sequence (A), shows that $\mathfrak{B}_{k}$ cannot exist for $k \geq 4$.

Theorem 4. $A$ ring spectrum $B_{2}$ does not exist.

Proof. The $E_{2}$-term of the Adams spectral sequence (A) is $\operatorname{Cotor}_{T(2)}\left(Z_{2}, Z_{2}\right)$, which is computed in [5, §3], where $T(2)$ is called $B$. In the notation of [5, §3], $P(0,1) \in E_{2}^{7,2}$ is a nonzero infinite cycle because $E_{2}^{8,0}=0$. Let $\rho \in \pi_{7} B_{k}$ project to $P(0,1)$. In $E_{2}^{14,3}, h_{0} P(0,1)^{2}=\Phi_{0}^{2} \Phi_{1} P(0,1) \neq 0$. Note $E_{2}^{15,0}=E_{2}^{15,1}=0, E_{2}^{15,2}=Z_{2}(P(0,2))$, and $E_{2}^{15,3}=Z_{2}\left(h_{0} P(0,2)\right)$. Observe that $P(0,2) \in\left\langle\Phi_{0}, h_{0}, \Phi_{2}\right\rangle$ in $E_{2}$ by [5, Theorem 4.3(1)]. Now $\Phi_{0}$ and $\Phi_{2}$ are infinite cycles because there are no elements whose product with $h_{0}$ is 0 in degrees 0 and $12 .\left\langle\Phi_{0}, 2, \Phi_{2}\right\rangle$ is defined because $\Phi_{0}, \Phi_{2}$ is the only nonzero element of degree one, thirteen, respectively. By [4, Theorem 8.1], $P(0,2)$ is an infinite cycle. Thus, $h_{0} P(0,1)^{2}$ is nonzero in $E_{\infty}^{14,5}$ and $2 \rho^{2} \neq$ 0 , contradicting the fact that $\pi_{*} B_{2}$ is a commutative graded ring. Therefore, $B_{2}$ cannot exist.

Recall that $H_{*} \mathrm{MSp}=Z_{2}\left[\xi_{n}^{4} \mid n \geq 1\right] \otimes \mathfrak{S}$, where $\mathfrak{S}$ is a polynomial algebra of $\mathfrak{A}^{*}$-primitive elements.

Lemma 5. There is a map of spectra $f: B_{3}^{(24)} \rightarrow \mathrm{MSp}$ such that

(a) $f \mid B_{3}^{(0)}=\mu: S \rightarrow \mathrm{MSp}$;

(b) $f_{*}(\xi) \equiv \xi$ modulo the ideal spanned by $\overline{\mathfrak{S}}$ for all $\xi \in H_{n} B_{3}$ with $n<24$.

Proof. (a) We construct $f$ on $B_{3}^{(q)}$ by induction on $q \geq 0$. Let $f \mid B_{3}^{(0)}=\mu$. Note that $H_{*} B_{3}$ is nonzero only in degrees divisible by eight. Assume that $f$ has been defined on the $8(t-1)$-skeleton of $B_{3}, 1 \leq t \leq 3$. By [3, Lemma 
VI.3.2], the obstruction to extending $f$ to the $8 t$-skeleton of $B_{3}$ is an element of $H^{*}\left(B_{3} ; \pi_{8 t-1} \mathrm{MSp}\right)$. However, the first nonzero element of $\pi_{r} \mathrm{MSp}$ in a degree congruent to $3 \bmod 4$ occurs when $r=31$. Thus, we can extend $f$ to the 24-skeleton of $B_{3}$.

(b) If $\xi \in H_{n} B_{3}, n \leq 23$, then $(1 \otimes \varepsilon) \psi f_{*}(\xi)=(1 \otimes \varepsilon)\left(1 \otimes f_{*}\right) \psi(\xi)=$ $(1 \otimes \varepsilon) \psi(\xi)=\xi \otimes 1$. Kernel $(1 \otimes \varepsilon) \psi$ equals the ideal spanned by the $\mathfrak{A}^{*}$ primitive elements of positive degree of $H_{*} \mathrm{MSp}=Z_{2}\left[\xi_{n}^{4} \mid n \geq 1\right] \otimes \mathfrak{S}$, which is $\overline{\mathfrak{S}}$. Thus, $f_{*}(\xi) \equiv \xi$ modulo the ideal spanned by $\overline{\mathfrak{S}}$.

Theorem 6. $A$ ring spectrum $B_{3}$ does not exist.

Proof. Observe that ${ }_{M} E_{1}^{11,1}=Z_{2}\left(h_{22}\right)={ }_{M} E_{\infty}^{11,1}$ because ${ }_{M} E_{1}^{12,0}=0$. Thus, $E_{2}^{11,1}=Z_{2}(R)$. A straightforward calculation shows that ${ }_{M} E_{2}^{10, k}=0$ for $k \geq 3, k \neq 6$, and that ${ }_{M} E_{2}^{10,6}=Z_{2}\left(h_{11}^{2} h_{20}^{4}\right)$. Thus, the only possibility for a nonzero differential on $R$ is $d_{5}(R)=h_{1}^{2} Q$, where $h_{1}, Q$ is represented by $h_{11}, h_{20}^{4}$, respectively, in the May spectral sequence. Let $f: B_{3}^{(24)} \rightarrow$ MSp denote the map of Lemma 5. Then $f_{*}\left(h_{1}^{2} Q\right)=\eta^{2} q_{0} \neq 0$ in $\pi_{10} \mathrm{MSp}$. Thus, $R$ is an infinite cycle. Let $\lambda \in \pi_{11} B_{3}$ project to $R$. Note that $0 \neq h_{10} h_{22}^{2} \in{ }_{M} E_{1}^{22,3}$ is an infinite cycle. Since ${ }_{M} E_{1}^{23, k}=0$ for $0 \leq k \leq 2,0 \neq h_{0} R^{2} \in E_{2}^{22,3}$ and $E_{2}^{23,0}=E_{2}^{23,1}=0$. Therefore, $h_{0} R^{2}$ is a nonbounding infinite cycle. Thus, $2 \lambda^{2} \neq 0$, which contradicts the fact that $\pi_{*} B_{3}$ is a commutative graded ring. Therefore, $B_{3}$ cannot exist.

\section{BIBLIOGRAPHY}

1. J. F. Adams, On the non-existence of elements of Hopf invariant one, Ann. of Math. (2) 72 (1960), 20-104.

2. E. H. Brown and F. P. Peterson, $A$ spectrum whose $Z_{p}$ cohomology is the algebra of reduced p-th powers, Topology 5 (1966), 149-154.

3. S. T. Hu, Homotopy theory, Pure Appl. Math., vol. 8, Academic Press, New York, 1959.

4. S. O. Kochman, A chain functor for bordism, Trans. Amer. Math. Soc. 239 (1978), 167-196.

5. __ The symplectic cobordism ring, I. Mem. Amer. Math. Soc., no. 228, Amer. Math. Soc., Providence, RI, 1980.

6. __ The symplectic Adams-Novikov spectral sequence for spheres, submitted.

7. A. Liulevicius, Notes on homotopy of Thom spectra, Amer. J. Math. 86 (1964), 1-16.

8. J. P. May, The cohomology of restricted Lie algebras and of Hopf algebras; application to the Steenrod algebra, Ph.D. thesis, Princeton Univ., 1964.

9. $\ldots$, The cohomology of restricted Lie algebras and of Hopf algebras, J. Algebra 3 (1966), 123-146.

10. J. W. Milnor and J. C. Moore, On the structure of Hopf algebras, Ann. of Math. 81 (1965), 211-264.

Department of Mathematics, York University, 4700 Keele Street, North York, ONTARIO M3J 1 P3 CANADA 